\title{
Double-Field Hadrontherapy Treatment Monitoring with the INSIDE In-Beam PET Scanner: Proof of Concept
}

\author{
Veronica Ferrero, Member, IEEE, Maria Giuseppina Bisogni, Niccolo’ Camarlinghi, Elisa Fiorina, \\ Giuseppe Giraudo, Matteo Morrocchi, Francesco Pennazio, Giancarlo Sportelli, Member, IEEE, Richard Wheadon \\ and Piergiorgio Cerello
}

\begin{abstract}
Positron Emission Tomography (PET) is a well established imaging technique for range monitoring in hadrontherapy. Multiple fields delivery is a standard protocol in treatments, but because of washout and residual activity background from previous irradiation plans, to this date a quantitative verification of the particle range for each beam field is still an open issue. In this paper, the proof of concept of a new method to evaluate with a PET detector the activity range of the second field of a treatment is discussed.

A proton and a carbon ion treatment plan with two parallelopposed beam fields were delivered on PMMA phantoms. In both cases, the second beam field was extracted from the first irradiation residual activity and compared to a reference image, obtained from the experimental acquisition of the second field alone.

Results demonstrate good agreement between the extracted second field and the reference image, with average difference in the activity range along the preferential direction of the beam less than $0.5 \mathrm{~mm}$ for protons, and $1.5 \mathrm{~mm}$ for carbon ions. Without taking into account any preferential direction, differences within $0.5 \mathrm{~mm}$ were found for both cases.

The method will soon be tested with non-homogeneous phantoms and, successively, also with in-vivo clinical data.
\end{abstract}

Index Terms-Radiation Therapy, Clinical Imaging System, Positron Emission Tomography, Monitoring, Image Analysis

\section{INTRODUCTION}

Because of its characteristic dose deposition profile as a function of depth, hadrontherapy allows highly conformal dose distribution and sparing of organs at risk. However, the steep dose gradient at the distal edge of the Bragg peak makes treatments sensitive to range uncertainties, which can lead to tumor under-dosage or unwanted radiation to healthy tissues. Range uncertainties can derive from Hounsfield Unit (HU) conversion methods, patient mispositioning, organ motion or anatomical changes during the course of the treatment, preventing the full exploitation of hadrontherapy physics advantages. In clinical practice, robustly optimized conservative

V. Ferrero (e-mail: veronica.ferrero@unito.it) is with the University of Torino, Physics Department, Torino, Italy.

V. Ferrero, P. Cerello, E. Fiorina, G. Giraudo, F. Pennazio and R. Wheadon are with the INFN Sezione di Torino, Torino, Italy.

M.G. Bisogni, N. Camarlinghi, M. Morrocchi and G. Sportelli are with the University of Pisa, Physics Department and INFN Sezione di Pisa, Pisa, Italy.

This project was funded by the Italian Ministry of Education under the national research program PRIN MIUR 2010P98A75 from 2013 to 2016, the Italian Institute of Nuclear Physics (INFN) and, from 2017, the CNAO Foundation. plans are used to account for these uncertainties, with (2.5 - 3.5)\% + (1 - 3) mm safety margins applied to the tumor volume [1]. Nowadays, in-vivo range verification is one of the most promising approaches to overcome these limitations. Over the last decades, various monitoring techniques have been developed, based on the detection of secondary particles generated from beam-tissues nuclear interactions, such as prompt photons, charged fragments, and $\beta^{+}$emitters [2]-[8]. A well established non-invasive technique for in-vivo range verification is Positron Emission Tomography (PET) which measures the $\beta^{+}$activity by detecting the back-to-back 511 $\mathrm{keV}$ photon pairs coming from positron annihilation [6], [9]-[12]. The $\beta^{+}$emitters can derive from both tissue and projectile fragmentation for heavy ion irradiation, or tissue fragmentation only for proton irradiation. Due to the tissue composition, the $\beta^{+}$emitters produced during irradiation are mostly carbon and oxygen isotopes. These radionuclides have short half lives (the longest being the ${ }^{11} \mathrm{C}$, whose half life is about $20 \mathrm{~min}$ ), making real time imaging essential. In order to minimize the biological washout effects and obtain the highest correlation between the measured activity and the deposited dose, the $\beta^{+}$decaying signal must be acquired while the treatment is ongoing [13]. This technique is called in-beam PET.

During 2016 a new in-beam PET scanner named INSIDE (Innovative Solutions for In-beam Dosimetry in hadronthErapy) was installed at the Italian Center of Oncological Hadrontherapy (CNAO) in Pavia, Italy, and characterized with PMMA phantoms [8], [14]-[16]. In December 2016, the in-beam PET was tested clinically, monitoring in real time the first irradiation of a two-field proton treatment plan of a patient [11].

When designing a treatment plan, multiple fields are standard protocol to optimise the dose to the target and minimize the dose delivered to the surrounding healthy tissues and organs at risk. Several groups monitored the PET activation during or after the treatment delivery in a clinical setting, with patients undergoing either one or two beam fields [6], [9]-[11], [17], [18]. Range monitoring in presence of more than one beam field introduces additional challenges due to the superposition of the activity distributions, along with the issues of washout and low activity levels, especially if data is acquired after the treatment with the scanner either positioned inside the treatment room (in-room PET) or in a dedicated room (off- 
room PET). These limitations can be overcome by the use of an in-beam PET scanner.

Previous studies report range monitoring analysis about the overall activation after the delivery of the whole treatment, but without focusing on the single contribution of each field [6], [17], [18]. Discerning the activity of subsequent beam fields would allow quality control not for just one beam field, but for the whole treatment session.

In this work, we present a novel method based on event rate selection and image filtering to extract consecutive beam fields acquired with the INSIDE in-beam PET. To this purpose, two clinical treatment plans were randomly selected from the CNAO database: one with protons, the other with carbon ions. Each of the selected plans consisted of two parallel-opposed beam fields, i.e., with the beams at an angle of $180^{\circ}$, so as to have the distal edge of the activity from the first beam inside the signal of the second one. This kind of configuration has never been explored before in PET-based monitoring, since the distal edge was considered undetectable because of the blurring of one beam into the other [18]. The activity of the second field was then extracted from the first one, and its distribution compared to a reference experimental distribution. Range analysis results are reported.

The paper is organized as follows. In Section II the INSIDE in-beam PET scanner characteristics, experimental setup and data acquisition are described. In Section III, the second field activity extraction analysis is presented. Section IV outlines the image comparison methods and provides the results of the analysis. Finally, the conclusions are drawn in Section V.

\section{INSIDE IN-BEAM PET EXPERIMENTAL SETUP AND ACQUISITION}

\section{A. The INSIDE In-Beam PET Scanner}

The scanner is based on two opposite planar heads consisting of $2 \times 5$ detection modules each. A detection module has $16 \times 16$ segmented Lutetium Fine Silicate (LFS) scintillating crystals, each $3 \times 3 \times 20 \mathrm{~mm}^{3}$, coupled 1:1 to Hamamatsu Multi-Pixel Photon Counters (MPPCs), resulting in $2560^{2}$ possible Lines Of Response (LOR) within the Field Of View (FOV). The module area is $51.2 \times 51.2 \mathrm{~mm}^{2}$. The total detection area for each head is of $112 \mathrm{~mm}$ (transaxial) $\times 264$ $\mathrm{mm}$ (axial, parallel to the direction of the beam).

The energy and detection time of the acquired events are measured by the 64-channel TOFPET ASIC [19]. An FPGA-based data processing system selects the events around the $511 \mathrm{keV}$ photopeak and transmits them to a Data Acquisition System (DAQ) implemented on a server (32 Hyper Threaded cores and 128 GB RAM) in which a high performance software applies online data sorting and writes time-tagged coincidence data. A Maximum Likelihood Expectation Maximization (MLEM) algorithm with 5 iterations is used to reconstruct time-resolved activity images $\left(224 \times 112 \times 264 \mathrm{~mm}^{3}\right.$ FOV with $1.6 \times 1.6$ $\times 1.6 \mathrm{~mm}^{3}$ voxel size). A more detailed description of the system is reported in [8].

\section{B. Experimental Setup}

The in-beam PET scanner is mounted on a movable cart, which was manually positioned and aligned with the treatment room lasers. The working distance between the heads was set at $50 \mathrm{~cm}$.

The irradiation plans reported in this work presented a dynamically shaped Spread-Out-Bragg-Peak (SOBP). Data were acquired at the CNAO synchrotron facility in Pavia, Italy, where treatment delivery is performed by an active scanning system. The selected plans were delivered to two $15 \times 15 \times 20 \mathrm{~cm}^{3}$ PMMA homogeneous phantoms. Both plans consisted of two beam fields, the second of which was delivered on the same phantom, after a $180^{\circ}$ rotation around the isocenter, so that the first beam field residual activity resulted superimposed to the second in the distal fall-off area. The time between the two irradiation deliveries was constrained by the time necessary to rotate the phantom and request the new treatment delivery to the synchrotron control room, but it was compatible with the typical time interval necessary in clinics to rotate the patient bed for the new irradiation field.

\section{Data Acquisition}

The CNAO synchrotron presents a pulsed beam structure comprising actual beam delivery (in-spill) followed by a pause (inter-spill). At the moment of the acquisition, the accelerator duty cycle was $3 \mathrm{~s}$, with about $1 \mathrm{~s}$ in-spill and 2 $\mathrm{s}$ inter-spill.

The DAQ system provides online selection of in/inter-spill coincidences, allowing quasi real-time reconstruction of both data sets. Data is thus acquired during both in- and inter-spill periods but, because of prompt radiation and neutron background during the spill, only inter-spill data is considered relevant and is used for the analysis [20].

Since data is time-tagged, specific time intervals can be selected and reconstructed. Both inter-spill and after-treatment data were taken into account for image reconstruction. All acquired inter-spill periods were considered. After-treatment data was acquired between the two beam fields delivery and for $145 \mathrm{~s}$ after treatment end.

The coincidence event rate, showing the in- and inter-spill distribution for the proton treatment delivery is reported in Fig. 1. Three time intervals are highlighted. Data from these time intervals is used to reconstruct the images shown in Fig. 2 - specifically: the period labelled as Field 1 (a) comprises both data from the treatment and after-treatment of the first beam field delivery, before phantom rotation; the Field 1 decay $(b)$ period refers to the sole decay data, after the phantom rotation and before the second field irradiation; finally, Field $2(c)$ indicates data comprising the second beam delivery and $145 \mathrm{~s}$ after-treatment data. The Field 2 activity image thus results on the opposite side of the phantom with respect to the isocenter when compared with the activity image acquired in Field 1, with one distal edge blurred inside the other. The same procedure was applied for the carbon ion case.

The maximum coincidence event rate detected for the proton and carbon ion treatment plan was of 19 and 3 $\mathrm{kHz}$, respectively, with a coincidence time window set to 2 
TABLE I

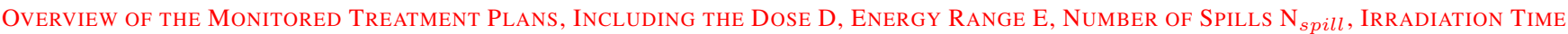
$\mathrm{T}_{\text {irr }}$ AND PET ACQUisition TIME $\mathrm{T}_{P E T}$.

\begin{tabular}{ccccccc}
\hline \hline & & $\mathrm{D}(\mathrm{GyE})$ & $\mathrm{E}(\mathrm{MeV} / \mathrm{u})$ & $\mathrm{N}_{\text {spill }}$ & $\mathrm{T}_{\text {irr }}(s)$ & $\mathrm{T}_{\text {PET }}(s)$ \\
\hline \multirow{3}{*}{ Proton Beam } & Field 1 & 1.9 & $62-129$ & 46 & 130 & 184 \\
& Field 1 decay & $/$ & $/$ & $/$ & $/$ & 180 \\
& Field 2 & 1.1 & $83-150$ & 53 & 169 & 314 \\
\hline \multirow{3}{*}{ Carbon Ion Beam } & Field 1 & 2.05 & $134-269$ & 45 & 163 & 214 \\
& Field 1 decay & $/$ & $/$ & $/$ & $/$ & 159 \\
& Field 2 & 2.05 & $134-264$ & 48 & 181 & 326 \\
\hline
\end{tabular}

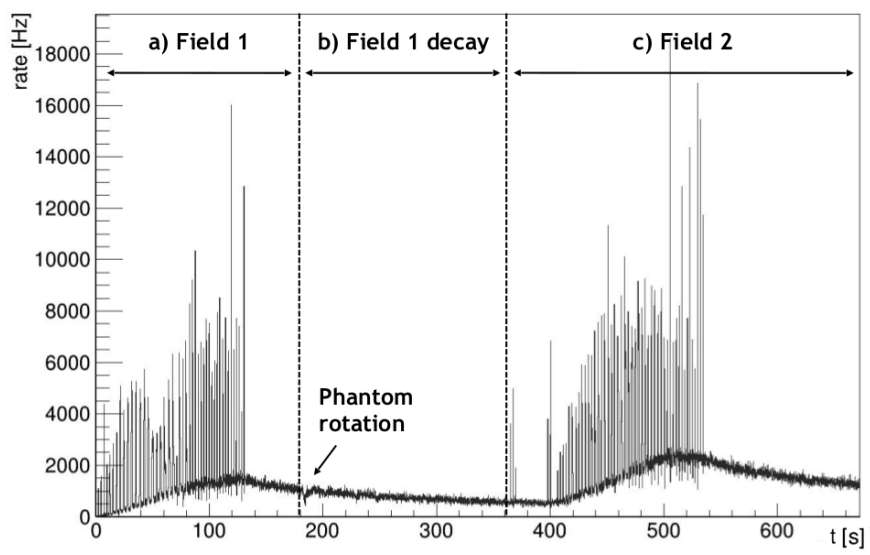

Fig. 1. Coincidence event rate of a treatment plan with parallel-opposed beam fields, protons. The time intervals used for the analysis are highlighted in the figure. a) Field 1: first beam field delivery and after-treatment data before the rotation of the phantom. b) Field 1 decay: after-treatment data of Field 1 after the rotation of the phantom. c) Field 2: second beam field delivery and after-treatment data.

ns. The total number of coincidences acquired during the inter-spill and after-treatment of Field 1 was of about $1.410^{5}$ for the proton treatment. The same order of magnitude of coincidences was observed for the in-vivo case monitored by the INSIDE scanner, with minor numerical discrepancies due to differences in phantom/patient attenuation and treatment plans [11]. For Field 1 of the carbon ion treatment, $310^{4}$ coincidences were acquired.

The total irradiation time and PET scan acquisition times used to reconstruct the images are reported in Table I for the selected cases. The number of observed spill, dose and energy range are also shown.

\section{SECOnd Field ACtivity Extraction}

The aim of this study is the extraction and validation of the second beam field activity distribution.

Fig. 2 shows the 2D images of the activity induced respectively by the proton (upper row) and carbon ion (lower row) treatment plans reconstructed considering the selected time intervals. The time intervals comprise both the actual delivery of the beam and the after-treatment data, as reported in Section II-C. The first field is shown before $(a)$ and after $(b)$ the rotation of the phantom, whereas the following irradiation $(c)$ starts from the beginning of the second field, so as to have as less residual activity as possible, and comprises $145 \mathrm{~s}$ aftertreatment for both treatments.

Field 1 can be straightforwardly compared to a reference image, originating either from a Monte Carlo simulation or another experimental acquisition. Monte Carlo simulations of the proton beam treatment plan, in particular, have already been discussed and validated in a previous work [16]. The carbon ion treatment plan simulation validation with experimental data is also ongoing [21].

In order to evaluate the contribution of Field 2 during the acquisition time interval $c$, in which the total distribution is given by the superposition of both beam fields coincidence event rates, the activity background given by the decay of Field 1 has first to be assessed. The proposed method goes as follows:

- The Field 1 contribution (i.e., the number of coincidences) in the time interval $c$ is evaluated by extrapolating the trend of the event rate decay curve found during $b$ with an exponential function.

- The Field 1 contribution should be translated into an activity image, useful to isolate Field 2 in the time interval $c$.

- The calculated number of coincidences of Field 1 is then used to select, at end of the $b$ data set (ordered in time), the coincidences to reconstruct this image, so as to be as close as possible to the Field 1 activity distribution expected in the $c$ data set.

- The obtained image represents the Field 1 decay background and is subtracted to the overall activity distribution acquired during the time interval $c$.

- The final image of Field 2 is thus obtained and compared to a reference image.

The reference consists of data acquired during an additional experiment, in which only Field 2 was delivered. The reproducibility of activity distributions delivered on PMMA phantoms was already studied in previous works and repeated experimental data can be thus considered as reliable reference images for a comparison analysis [15], [16].

The distribution of the second field devoid of the previous activity background is shown in Fig. $3(a)$, along with the reference image $(b)$ for the proton (upper row) and carbon ion (lower row) treatment plans. As it can be seen from both Fig. 2 and 3, the carbon ion images present more noise, due 

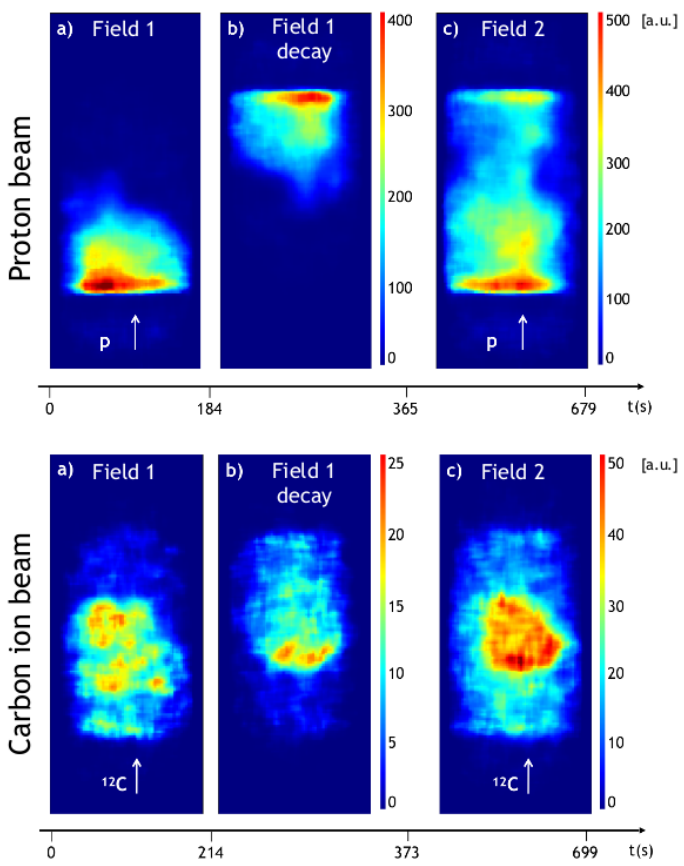

Fig. 2. Reconstructed 2D images of the induced activity: central slice parallel to the PET heads for different time-intervals for the proton (upper row) and the carbon ion (lower row) treatment. a) Field 1 irradiation. b) Decay of Field 1 after a rotation of the phantom of $180^{\circ}$ around the isocenter. c) Field 2 with residual activity from the previous irradiation. The beam direction is shown in the images. The images refer to different intensity scales because of the different statistics and integration time intervals.

to their characteristic tail given by the charged fragments, and also their lower statistics compared to protons. Indeed, treatments with carbon ions have a number of primary particles which is typically an order of magnitude of 10 lower than that of protons. In particular, when subtracting images with uncorrelated statistics (Fig. 3), this translates into higher noise in the final image that has to be excluded when performing the range assessment analysis.

The methods used to compare the images are presented in the next section.

\section{ACTIVITY COMPARISON}

\section{A. Image Processing}

The analysis was implemented in C++ with ROOT $^{1}$ and Insight Segmentation and Registration Toolkit ${ }^{2}$ (ITK) libraries, and allows selection and reconstruction of specific time intervals, yielding time-activity evolution comparisons.

The analysis relies on the extraction of an iso-activity surface $S$ from each PET image through the application of image filters. First, a median filter with a $5 \times 5 \times 5 \mathrm{~mm}^{3}$ kernel is applied to the PET images to compensate for the salt and pepper noise related to the image statistics. Then, the images are equalized to mitigate the contribution of small intensity variations and a $20 \%$ threshold filter with respect to the maximum image intensity is applied to exclude the noise below that threshold. Erosion and dilation filters are

\footnotetext{
${ }^{1}$ https://root.cern.ch

${ }^{2}$ https://itk.org
}

also applied to exclude eventual noise spots left and obtain fully-connected activity surfaces. Fig. 4 shows the activity profiles, normalized to the maximum intensity of the image, of the extracted Field 2. The profiles are calculated along the beam axis $z$, considering a square area of $0.16 \times 0.16 \mathrm{~cm}^{2}$ (corresponding to 1 voxel) in the transverse plane from the center of the image. As it can be seen from the image, voxel intensities below $20 \%$ with respect to the maximum value are excluded in the analysis.

Further filtering is done by integrating the spatial information of the CNAO dose delivery system, so that only the voxels through which the beam is actually delivered are taken into consideration [22]. The resulting surface $S$ and the reference (shown in Fig. 3) are then compared. The range analysis has been tested for clinical treatments delivered on PMMA phantoms and, more recently, to assess the measured activity compliance with the expected distribution in a clinical in-vivo treatment [8], [11], [16], [21], [23].

Previous studies report activity comparisons along the beam direction only, or by visual means performed by specialized personnel [10], [17], [24]-[26]. In this work the analysis was carried out taking into account the whole 3-dimensional activity distribution both in the sole direction of the beam and in all directions. The first method was designed to maximize the sensitivity of the scanner in detecting range differences along the beam direction, whereas the second method was designed to give an insight about the absolute distance between the two distributions in space. Both methods provide quality indicators of the agreement between the two images. The comparison methods are described in sections IV-B and IV-C.

\section{B. Beam's Eye View (BEV)}

The images are compared by evaluating, in the preferential direction of the beam, the difference between the entrance and exit points of the surface $S$, which is considered to be the activity range $R$. The activity ranges $R$ and $R_{\text {reference }}$ of the two images are then subtracted and the average range difference $\langle\delta R>$ and the distribution FWHM are considered as the comparison quality indicators.

\section{Overall View $(O V)$}

The images are compared by evaluating, for each voxel belonging to $S$, the minimum Euclidean distance $\delta S$ between the two iso-activity surfaces, with no preferential direction. The average surface distance $\langle\delta S\rangle$ and the FWHM are considered as the comparison quality indicators.

\section{Results}

Fig. 5 shows the range difference (left) and surface distance (right) distributions obtained comparing the images of the second field shown in Fig. 3.

The BEV method indicates an agreement of $6.4 \mathrm{~mm}$ FWHM (protons) and $11.2 \mathrm{~mm}$ FWHM (carbon ions), with minimal systematic effects: the average range differences $\langle\delta R\rangle$ are $0.23 \mathrm{~mm}$ and $1.28 \mathrm{~mm}$ for the proton and carbon ion case, respectively. The OV method shows $1.6 \mathrm{~mm}$ FWHM and 

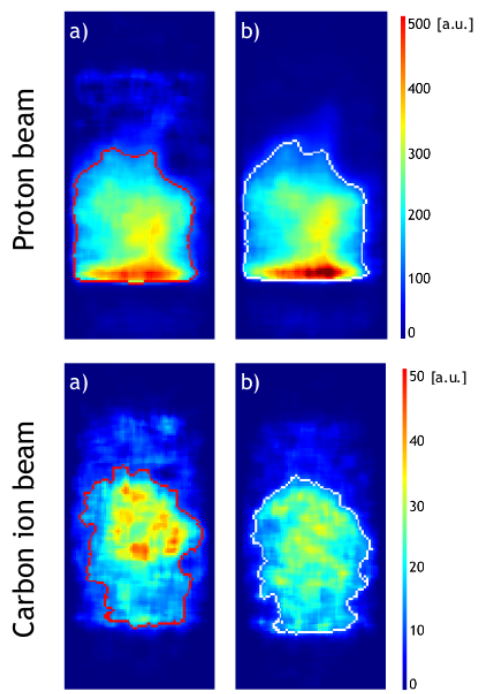

Fig. 3. a) The $2 \mathrm{D}$ activity image obtained subtracting the previous irradiation background from the second field image. b) Activity image acquired during the irradiation of Field 2 alone for a proton (upper row) and carbon ion (lower row) treatments. The iso-activity surfaces $\mathrm{S}$ are highlighted in red for the extracted second field, and white for the single acquisition. The images refer to different intensity scales because of the different statistics and integration time intervals. a)

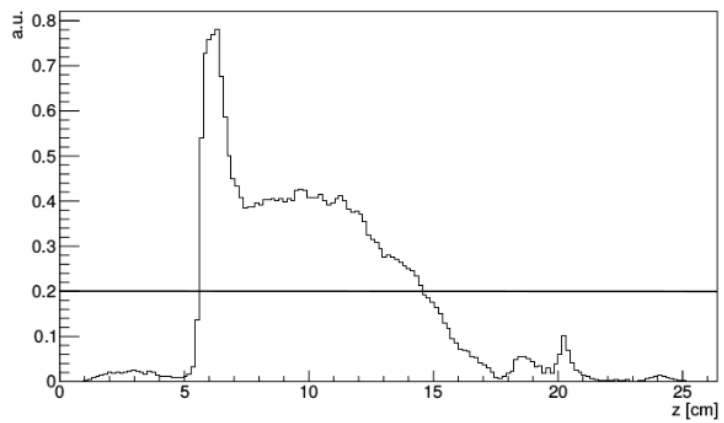

b)

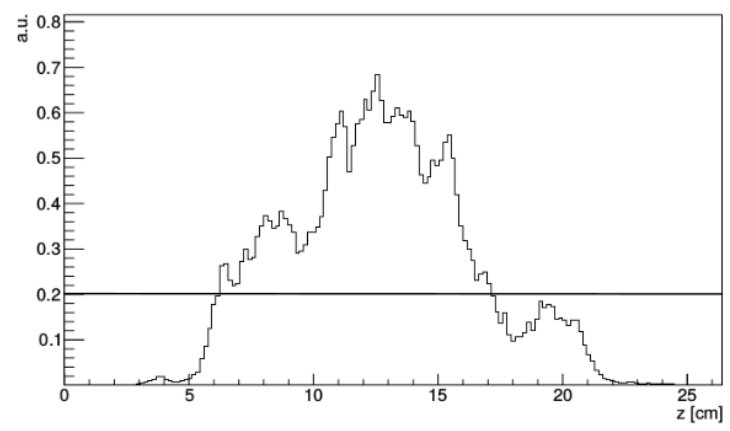

Fig. 4. a) Proton beam and b) carbon ion beam activity profiles of the extracted Field 2, normalized to the maximum intensity value of the image. A line representing the $20 \%$ threshold, below which intensities are excluded, is also shown.

average surface distance $\langle\delta S>$ of $0.22 \mathrm{~mm}$ for protons, and $4.8 \mathrm{~mm}$ FWHM and $\langle\delta S>$ of $0.33 \mathrm{~mm}$ for carbon ions. Both methods present slightly worse agreements for the carbon ion case because of its reduced statistics, which makes the range evaluation less precise.

The activity images were further compared through a Pearson
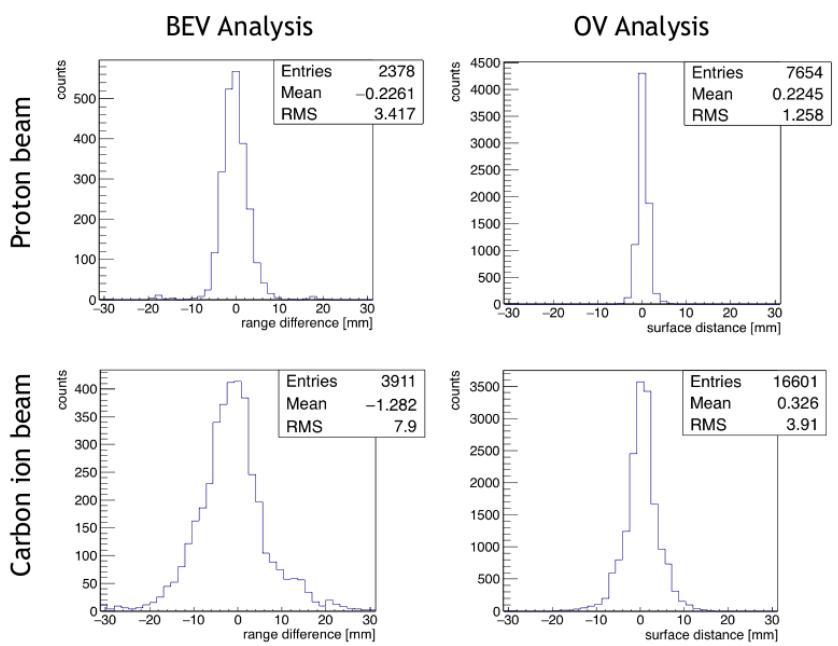

Fig. 5. Beams eye view (BEV) average range difference and overall view (OV) surface distance distributions for a proton (upper row) and carbon ion (lower row) treatment plan comparison between the extracted second field and the reference distribution of the same field. Mean and standard deviation (RMS) of the distributions are enclosed in the figure.

correlation test as described in [27]. The test gives a numerical value between $[0,1]$, stating how strong a correlation can be found between the image intensities. The resulting values were 0.98 and 0.92 for the proton and carbon ion case, respectively, showing strong correlation.

Moreover, the same analysis methods were applied to compare the first beam field with a reference experimental activity image, so as to have a reliable comparison with the quality indicators values. That is, for both treatments the first beam field was again delivered on a PMMA phantom and its corresponding activity image, reconstructed within the same time interval as indicated by the Field 1 notation, was used as reference.

Results show that the comparison between the Field 1 image with its reference have quality indicators compatible with the ones found in the comparison between the extracted second field image and its reference, for both the proton and carbon ion case. Since the Field 1 images were not post-processed to remove any activity background coming from a previous irradiation, and data reproducibility is considered as reliable, the obtained comparable quality indicators validate the proof of concept presented in this work to monitor consecutive beam fields.

A summary of the analysis quality indicators is reported in Table II. The Field 1 and Field 2 notation refer to the experimental images at comparison.

\section{Conclusions}

The activity distribution of a clinical treatment with two parallel-opposed beam fields was measured with the INSIDE in-beam PET scanner for a proton and a carbon ion treatment plan. Despite the residual activity due to the first irradiation that was partially superimposed to the second field activity distribution, the activity produced solely by the second beam 
TABLE II

VALUES OF THE QUALITY INDICATORS OF THE COMPARISON ANALYSIS.

\begin{tabular}{lccccc}
\hline \hline \multirow{2}{*}{ BEV Analysis } & \multicolumn{2}{c}{ Proton Beam } & \multicolumn{2}{c}{ Carbon Ion Beam } \\
& Range difference $\langle\delta R>$ & $0.34 \mathrm{~mm}$ & $0.23 \mathrm{~mm}$ & $2.29 \mathrm{~mm}$ & $1.28 \mathrm{~mm}$ \\
& FWHM & $4.8 \mathrm{~mm}$ & $6.4 \mathrm{~mm}$ & $9.6 \mathrm{~mm}$ & $11.2 \mathrm{~mm}$ \\
\multirow{2}{*}{ OV Analysis } & Surface distance $\langle\delta S>$ & $0.11 \mathrm{~mm}$ & $0.22 \mathrm{~mm}$ & $0.38 \mathrm{~mm}$ & $0.33 \mathrm{~mm}$ \\
& FWHM & $1.6 \mathrm{~mm}$ & $1.6 \mathrm{~mm}$ & $4.8 \mathrm{~mm}$ & $4.8 \mathrm{~mm}$ \\
\hline
\end{tabular}

field was extracted with a novel method and compared to an experimental reference image. This allowed, to out knowledge for the first time, to conduct a range assessment analysis on both fields, separately.

The range comparison was carried out taking into account both the sole direction of the beam (BEV method) and all directions (OV method). Results show a $6.4 \mathrm{~mm}$ FWHM and a $11.2 \mathrm{~mm}$ FWHM in range difference distribution (BEV), with average difference along the beam direction less than $0.5 \mathrm{~mm}$ for protons, and $1.5 \mathrm{~mm}$ for carbon ions. Without taking into account any preferential direction, $1.6 \mathrm{~mm}$ FWHM and $4.8 \mathrm{~mm}$ FWHM in surface distance distribution (OV) are found, with differences within $0.5 \mathrm{~mm}$ for both cases. The values obtained from the OV method show that there is a good agreement between the whole 3-dimensional activity distributions at comparison. The wider BEV distributions are due to the fact that there is a preferential direction to be considered, which makes the definition of the range near the activity borders more critical, especially for the carbon ion case, where the statistics is a factor of ten lower than in the proton case (see Fig. 3). Nevertheless, the Pearson correlation test shows a strong intensity correlation between the images at comparison.

The obtained quality indicators are similar to the ones obtained for the comparison between the Field 1 activity and its reference. Furthermore, they are comparable to the results obtained for the range assessment in an in-vivo clinical case, indicating the feasibility of the method.

This first attempt to measure multiple fields and assess the activity range of the second beam field with the same analysis performed on the first one poses very promising prospects for in-beam PET. The proof of concept will also be tested on nonhomogeneous phantoms.

The INSIDE in-beam PET will soon start a clinical trial at the CNAO facility that will include the monitoring of multiple fields treatment plans. The method presented here will then be tested with real patient treatment data, assessing its feasibility in a clinical environment, where a more complex response due to the contribution of washout and heterogeneous tissues is expected.

\section{REFERENCES}

[1] H. Paganetti, "Range uncertainties in proton therapy and the role of monte carlo simulations," Physics in Medicine and Biology, vol. 57, no. 11, pp. R99-R117, 2012.

[2] A. C. Knopf and A. Lomax, "In vivo proton range verification: a review," Physics in Medicine and Biology, vol. 58, p. R131R160, 2013.
[3] M. Durante and H. Paganetti, "Nuclear physics in particle therapy: a review," Reports on Progress in Physics, vol. 79, no. 9, 2016.

[4] J. Krimmer, D. Dauvergne, J. Létang, and E. Testa, "Prompt-gamma monitoring in hadrontherapy: A review," Nuclear Instruments and Methods in Physics Research Section A: Accelerators, Spectrometers, Detectors and Associated Equipment, 2017. [Online]. Available: https://hal.archives-ouvertes.fr/hal-01585334

[5] S. Muraro, G. Battistoni, F. Collamati, E. De Lucia, R. Faccini, F. Ferroni, S. Fiore, P. Frallicciardi, M. Marafini, I. Mattei, S. Morganti, R. Paramatti, L. Piersanti, D. Pinci, A. Rucinski, A. Russomando, A. Sarti, A. Sciubba, E. Solfaroli-Camillocci, M. Toppi, G. Traini, C. Voena, and V. Patera, "Monitoring of hadrontherapy treatments by means of charged particle detection," Frontiers in Oncology, vol. 6, p. 177, 2016. [Online]. Available: https://www.frontiersin.org/article/10.3389/fonc.2016.00177

[6] K. Parodi, H. Paganetti, H. A. Shih, S. Michaud, J. S. Loeffler, T. F. DeLaney, N. J. Liebsch, J. E. Munzenrider, A. J. Fischman, A. Knopf, and T. Bortfeld, "Patient study of in vivo verification of beam delivery and range, using positron emission tomography and computed tomography imaging after proton therapy," International Journal of Radiation Oncology Biology Physics, vol. 68, pp. 920-934, 2007.

[7] G. Sportelli, N. Belcari, N. Camarlinghi, G. A. P. Cirrone, G. Cuttone, S. Ferretti, A. Kraan, J. E. Ortuño, F. Romano, A. Santos, K. Straub, A. Tramontana, A. D. Guerra, and V. Rosso, "First full-beam pet acquisitions in proton therapy with a modular dual-head dedicated system," Physics in Medicine and Biology, vol. 59, no. 1, p. 43, 2014. [Online]. Available: http://stacks.iop.org/0031-9155/59/i=1/a=43

[8] M. G. Bisogni, A. Attili, G. Battistoni, N. Belcari, N. Camarlinghi, P. Cerello, S. Coli, A. Del Guerra, A. Ferrari, V. Ferrero, E. Fiorina, G. Giraudo, E. Kostara, M. Morrocchi, F. Pennazio, C. Peroni, M. A. Piliero, G. Pirrone, A. Rivetti, M. D. Rolo, V. Rosso, P. Sala, G. Sportelli, and R. Wheadon, "Inside in-beam positron emission tomography system for particle range monitoring in hadrontherapy," Journal of Medical Imaging, vol. 4, no. 1, p. 011005, 2017.

[9] W. Enghardt, P. Crespo, F. Fiedler, R. Hinz, K. Parodi, J. Pawelke, and F. Ponisch, "Charged hadron tumour therapy monitoring by means of pet," Nuclear Instruments and Methods in Physics Research Section A, vol. 525, no. 1-2, pp. 284-288, 62004.

[10] T. Nishio, A. Miyatake, T. Ogino, K. Nakagawa, N. Saijo, and H. Esumi, "The development and clinical use of a beam on-line pet system mounted on a rotating gantry port in proton therapy," International Journal of Radiation Oncology Biology Physics, vol. 76, no. 1, pp. 277-286, 2010.

[11] V. Ferrero, E. Fiorina, M. Morrocchi, F. Pennazio, G. Baroni, G. Battistoni, N. Belcari, N. Camarlinghi, M. Ciocca, A. Del Guerra, M. Donetti, S. Giordanengo, G. Giraudo, V. Patera, C. Peroni, A. Rivetti, M. D. R. Rolo, S. Rossi, V. Rosso, G. Sportelli, S. Tampellini, F. Valvo, R. Wheadon, P. Cerello, and M. G. Bisogni, "Online proton therapy monitoring: clinical test of a silicon-photodetector-based in-beam pet," Scientific Reports, vol. 8, no. 1, p. 4100, 2018. [Online]. Available: https://doi.org/10.1038/s41598-018-22325-6

[12] A. Del Guerra, N. Belcari, and M. Bisogni, "Positron Emission Tomography: Its 65 years," Nuovo Cimento Rivista Serie, vol. 39, pp. 155-223, Apr. 2016.

[13] G. Shakirin, H. Braess, F. Fiedler, D. Kunath, K. Laube, K. Parodi, M. Priegnitz, and W. Enghardt, "Implementation and workflow for pet monitoring of therapeutic ion irradiation: a comparison of in-beam, in-room, and off-line techniques," Physics in Medicine and Biology, vol. 56, no. 5, p. 1281, 2011. [Online]. Available: http://stacks.iop.org/0031-9155/56/i=5/a=004

[14] E. Fiorina, "An integrated system for the online monitoring of parti- 
cle therapy treatment accuracy," Nuclear Instruments and Methods in Physics Research Section A, vol. 824, pp. 198-201, 2016.

[15] M. Piliero, N. Belcari, M. Bisogni, N. Camarlinghi, P. Cerello, S. Coli, A. D. Guerra, V. Ferrero, E. Fiorina, G. Giraudo, E. Kostara M. Morrocchi, F. Pennazio, C. Peroni, G. Pirrone, A. Rivetti, M. Rolo, V. Rosso, G. Sportelli, and R. Wheadon, "First results of the inside in-beam pet scanner for the on-line monitoring of particle therapy treatments," Journal of Instrumentation, vol. 11, no. 12, p. C12011, 2016. [Online]. Available: http://stacks.iop.org/1748$0221 / 11 / \mathrm{i}=12 / \mathrm{a}=\mathrm{C} 12011$

[16] V. Ferrero, "The inside project: on-line monitoring and simulation validation with the in-beam pet scanner," Journal of Physics: Conference Series, vol. 841, no. 1, p. 012011, 2017. [Online]. Available: http://stacks.iop.org/1742-6596/841/i=1/a=012011

[17] C. H. Min, X. Zhu, B. A. Winey, K. Grogg, M. Testa, G. E. Fakhri, T. R. Bortfeld, H. Paganetti, and H. Shih, "Clinical application of in-room pet for in vivo treatment monitoring in proton radiotherapy," International Journal of Radiation Oncology, Biology, Physics, vol. 86, no. 1, pp. 183-189, 2013.

[18] J. Handrack, T. Tessonnier, W. Chen, J. Liebl, J. Debus, J. Bauer, and K. Parodi, "Sensitivity of post treatment positron emission tomography/computed tomography to detect inter-fractional range variations in scanned ion beam therapy," Acta Oncologica, vol. 56 , no. 11, pp. 1451-1458, 2017, pMID: 28918686. [Online]. Available: https://doi.org/10.1080/0284186X.2017.1348628

[19] M. D. Rolo, R. Bugalho, F. Gonalves, G. Mazza, A. Rivetti, J. C. Silva, R. Silva, and J. Varela, "Tofpet asic for pet applications," Journal of Instrumentation, vol. 8, no. 02, p. C02050, 2013. [Online]. Available: http://stacks.iop.org/1748-0221/8/i=02/a=C02050

[20] F. Pennazio, G. Battistoni, M. Bisogni, F. Cappucci, P. Cerello, A. D. Guerra, E. Fiorina, G. Giraudo, A. Kraan, A. Mairani, M. Morrocchi, R. Nicolini, C. Peroni, M. Piliero, G. Pirrone, P. Sala, and R. Wheadon, "Monte carlo simulations for in-beam pet monitoring in hadron-therapy," in Book of Abstracts (XIII Turku PET Symposium), University of Turku, May 24-27, 2014, p. 78.

[21] F. Pennazio, G. Battistoni, M. G. Bisogni, N. Camarlinghi, A. Ferrari, V. Ferrero, E. Fiorina, M. Morrocchi, P. Sala, G. Sportelli, R. Wheadon, and P. Cerello, "Carbon ions beam therapy monitoring with the inside in-beam pet," Physics in Medicine and Biology, 2018. [Online] Available: http://iopscience.iop.org/article/10.1088/1361-6560/aacab8

[22] S. Giordanengo, M. A. Garella, F. Marchetto, F. Bourhaleb, M. Ciocca, A. Mirandola, V. Monaco, M. A. Hosseini, C. Peroni, R. Sacchi, R. Cirio, and M. Donetti, "The cnao dose delivery system for modulated scanning ion beam radiotherapy," Medical Physics, vol. 42, no. 1, pp 263-275, 2015. [Online]. Available: http://dx.doi.org/10.1118/1.4903276

[23] E. Fiorina, V. Ferrero, F. Pennazio, G. Baroni, G. Battistoni, N. Belcari, P. Cerello, N. Camarlinghi, M. Ciocca, A. D. Guerra, M. Donetti, A. Ferrari, S. Giordanengo, G. Giraudo, A. Mairani, M. Morrocchi, C. Peroni, A. Rivetti, M. D. R. Rolo, S. Rossi, V. Rosso, P. Sala, G. Sportelli, S. Tampellini, F. Valvo, R. Wheadon, and M. Bisogni, "Monte carlo simulation tool for online treatment monitoring in hadrontherapy with in-beam pet: A patient study," Physica Medica, 2018. [Online]. Available: http://www.sciencedirect.com/science/article/pii/

[24] A. Knopf, K. Parodi, H. Paganetti, E. Cascio, A. Bonab, and T. Bortfeld, "Quantitative assessment of the physical potential of proton beam range verification with pet/ct," Physics in Medicine and Biology, vol. 53, no. 15, p. 4137, 2008. [Online]. Available: http://stacks.iop.org/0031-9155/53/i=15/a=009

[25] K. Frey, D. Unholtz, J. Bauer, J. Debus, C. H. Min, T. Bortfeld, H. Paganetti, and K. Parodi, "Automation and uncertainty analysis of a method for in vivo range verification in particle therapy," Physics in Medicine and Biology, vol. 59, no. 19, p. 5903, 2014. [Online]. Available: http://stacks.iop.org/0031-9155/59/i=19/a=5903

[26] F. Fiedler, D. Kunath, M. Priegnitz, and W. Enghardt, Online Irradiation Control by Means of PET. Berlin, Heidelberg: Springer Berlin Heidelberg, 2012, pp. 527-543.

[27] P. Kuess, W. Birkfellner, W. Enghardt, S. Helmbrecht, F. Fiedler, and D. Georg, "Using statistical measures for automated comparison of in-beam pet data," Medical Physics, vol. 39, no. 10, pp. 5874-5881, 2012. [Online]. Available: http://dx.doi.org/10.1118/1.4749962 